infants receiving a DRC increased from $70 \%$ to $84 \%$ during the COVID-19 pandemic, with no change in mean admission temperature.

Conclusions Our results support DRC as a safe and feasible concept. The DRC ideology empowers women, supports bonding and provides preterm infants with the magnitude of benefits associated with early maternal contact. The recent BAPM maternal breast milk toolkit refers to early contact with parents as a key step in optimising MBM for preterm babies: DRC is an effective strategy in implementing this. Our instability data highlights the importance of following the standard operating procedure and ensuring continual close monitoring during DRC. Examining the mid and longer-term outcomes for DRC neonates and a larger, multi-centre study would be useful contributors to support standardising DRC in practice.

\section{EXPOSURE TO MOBILE SCREEN DEVICES IN EARLY CHILDHOOD AND CHILDREN'S LANGUAGE DEVELOPMENT}

Luisa Moraes Teixeira, Helen Bedford. UK

\subsection{6/bmjpo-2021-RCPCH.153}

Background The use of mobile devices by young children has increased considerably over the last decade and especially after the COVID-19 pandemic. However, high-level evidence on whether the use of digital mobile devices can help or hinder the development of language in early childhood is still lacking. Exposure to mobile devices is a modifiable environmental factor and having a greater understanding of this issue is essential to better guide both policy and practice.

Objectives To investigate the extent to which mobile media exposure (smartphones and tablets) in early childhood (the first five years of life) affects the development of language in typically developing children.

Methods A systematic review (SR) was performed in July 2020 using the Medline, Embase, PsycINFO and Web of Science databases. The search was limited to the English and Portuguese languages and to the last ten years. Articles were reviewed against the inclusion and exclusion criteria. A comprehensive quality assessment tool was developed by combining the recommendations of three previously established guides and the internal and external validity of the selected studies were assessed qualitatively. A narrative approach was used to synthesise the data extracted.

Results The search strategy retrieved 4,443 articles of which 3 met the inclusion and exclusion criteria. The studies included were cross-sectional, published between 2019 and 2020. Their sample size ranged from 117 to 893 participants. The mean age of children varied around 18.7 and 54 months at the time of screen and language assessments. In all studies, boys accounted for approximately 54\% of the participants. Different tools were used to assess language and to measure screen time, although all studies assessed both expressive and receptive language. Results of the quality assessment showed that the overall quality rate for all studies was poor. Limitations of the studies were discussed, and confounding variables related to the child, the family and the pattern of mobile device use were analysed. Overall, findings were heterogeneous and placing a dichotomous label for the effects of mobile device use on children's language development was not possible.
Conclusions The findings from this SR show that based on the current literature, it is not possible to categorise the effects of mobile device exposure on children's language development as either beneficial or detrimental, since strong evidence addressing these potential associations is lacking and since there are many covariates around them. Longitudinal studies are needed to clarify cause-and-effect relations and to elucidate the long-term effects of such exposure. Moreover, in order to reduce the risk of information bias, future studies should assess language skills professionally and measure mobile media use electronically rather than rely on parents' reports. In addition, future research should also take into account the content children watch and the quality of parent-child interactions during mobile screen use. Multicentre and cross-cultural studies should be conducted so that the external validity of findings can be increased. Certainly, paediatric societies and policymakers should consider all these factors while issuing recommendations and guidelines.

\section{INTEGRATED ELECTRONIC APPOINTMENTS AND FOLLOW UP SYSTEM IN AMBULATORY CARE. SIMPLE IT SOLUTIONS TO COMPLEX PROBLEMS}

Arun Mahay, Aditya Raj, Fionnuala Ryan. UK

\subsection{6/bmjpo-2021-RCPCH.154}

Background The paediatric ambulatory care department has multiple functions such as administration of IV antibiotics, blood transfusions, urgent outpatient blood tests and clinical reviews. The patients coming into the paediatric ambulatory care unit were organised in a physical handwritten diary with MDT members manually writing down who was supposed to come into the unit.

This led to many issues:

- There was no specific follow up system for these patients. Certain blood tests take 24 hours or longer to come back and when these were missed serious incidents occurred.

- Patients would often be double booked to see the same doctor

- Often the unit had too many patients in it for a single day leading to long waits and lower patient satisfaction

- Patient identifiable information was often illegible in the book making it difficult to understand who was coming into the unit

- There wasn't any space in the book for writing the reasons a patient was coming in. This often led to confusion

We created a computerised system in order to deal with these problems.

Objectives The objectives of this project were:

1. To increase the amount and clarity of patient identifiable details

2. To ensure the reason for the appointments was clearer

3. To reduce the number of double bookings

4. To reduce the number of serious incidents from patients not being followed up correctly.

Methods The multidisciplinary team involved in the project entirely upheaved the appointment system and created a computerised appointments book using excel. We used various techniques to help reduce the number of errors in the input 
of data. For example, error messages would appear if appointments were double booked. We also had a built-in follow up system. This would highlight patients whose results hadn't been followed up which would also help prevent mistakes. This QI project had 5 cycles in which the system was finetuned, tutorials were given to members of the MDT and feedback was taken. This helped us promote a sustainable change and allowed us to troubleshoot any issues with the appointment system. After each cycle our objectives were measured quantitatively.

Results

1. We found that the number of patients with three pieces of patient identifiable data increased by $56 \%$ (patients with at least 2 pieces of identifiable details also increased).

2. The number of patients with clear reasons inputted for coming to the paediatric ambulatory care unit increased by $22 \%$.

3. The number of double bookings decreased by $20 \%$.

4. The number of patients who weren't followed up were also reduced (results went down from 4 serious incident form in the 6 month period before the computerised system to no serious incident forms in the 6 months after the system.

Conclusions This QI project showed that simple IT solutions can often lead to dramatic improvements in patient safety and better care. The reduction of human error is important for any paediatric department. Involving the multidisciplinary team in projects can lead to a more sustainable change and is necessary when creating changes to systems.

\section{PARENTAL PERCEPTION ON INHALER AND SMOKING AT HOME}

Sze Ka Ng, Sze Ka Ng, Florence Choi, Po King Ma. Hong Kong

\subsection{6/bmjpo-2021-RCPCH.155}

Background Fear of treatment of asthma is common amongst patients and their carers, and often leads to poor disease control and poor quality of life. Some carers preferred the use of complementary or alternative medicine, such as Chinese herbal medicine or supplement, to inhaled medications.

Objectives This paper aims to describe the perception of parents with asthmatic children on inhaler treatment and smoking at home.

Methods The clinical trial was carried out at an acute respiratory Paediatric unit in a public hospital in Hong Kong. The proposal of the clinical trial was registered in the Chinese Clinical Trial Registry, World Health Organization Organisation (ChiCTR1800019706) and was approval from the Ethic Committee review board was obtained parental consents were obtained. Fifty-six children aged 4-11 years were admitted to an acute paediatric unit for asthmatic attack during the study period from Oct 2018 to Jun 2019. Their parents were invited to participate in a semi-structured interview using open-ended questions, guided by several themes. The interview lasted 15 to 20 minutes. Content analysis was used to analyze the data collected from the interviews. All transcriptions were undertaken by the same investigator.

Results Four aspects were concluded.

Doubt about asthma diagnosis. According to the most updated concept, asthma is considered an allergic disease that can be controlled without primary symptoms, such as repeated coughing, wheezing and breathing difficulties.
However, many parents perceived asthma as a different entity from allergic airway. Some were unable to differentiate between asthmatic attack, upper respiratory tract infection and wheezing in pneumonia. With these misconceptions, parents would doubt the diagnosis of asthma and would default follow-up eventually.

Steroid phobia. Many parents expressed their concern on the adverse effects of corticosteroids on their children, especially for those who started treatment at an older age. They were worried that inhaled corticosteroids would do more harm than good. One parent even used the term 'steroid abuse' when referring to the regular use of steroid. Another parent had fear of oral ulcer development as a side effect of corticosteroid.

Perception on inhaler. There were several misconceptions on inhaler medications. Some parents perceived the long term use of bronchodilator would lead to asthma or persist the asthma symptoms. Some thought nebulizing medications were more effective than delivering medications via inhalers.

Smoking at home. Some parents believed indirect exposure to tobacco smoke at home would not affect the control of asthma in their children. Parents understood the harm of smoking at home but expressed their difficulty in asking the elder family member living with child to quit smoking.

Conclusions These qualitative data highlight the communication problem between healthcare professionals and patients and the misconceptions of asthma management. Firstly, the updated concept on allergic diseases such as asthma, was not well-publicized. Many parents could not accept their children being diagnosed with asthma, although they themselves had asthma or other allergic disease during their childhood. Furthermore, the concept of controlled asthma used by healthcare professionals and parents are often different. Lastly, third-hand smoking is a commonly overlooked issue.

\section{HEALTH-RELATED QUALITY OF LIFE OF RARE DISEASE PATIENTS AND CARE-GIVERS IN HONG KONG}

Yvette Nga Chung Ng, Brian Hon Yin Chung, Claudia Ching Yan Chung. Hong Kong

\subsection{6/bmjpo-2021-RCPCH.156}

Background A rare disease (RD) is a disease affecting less than one in 2,000 people. In Hong Kong, one in 67 people is living with one or more RDs. RD patients live with different degrees of life-long physical or intellectual disability and often require special care. Approximately $80 \%$ of the RDs are genetic in origin, in which some are with childhood onset. The paediatric RD patients constitute $35 \%$ of the whole $\mathrm{RD}$ population in Hong Kong.

Objectives To investigate the health-related quality of life (HRQoL) of patients and care-givers in the RD population in Hong Kong.

Methods This was a cross-sectional study between 25 April and 16 October 2020. The EuroQol 5-Dimension 3-Level (EQ-5D-3L), a generic preference-based patient-reported outcome measure instrument, was distributed to the RD patients and care-givers through $\mathrm{RD}$ patient groups to describe HRQoL. The five dimensions assessed were mobility, self-care, usual activity, anxiety/depression and pain/discomfort. The three levels represent severity in each dimension, with Level 1 indicating absence of problem and Level 3 indicating extreme 